ZS Research Square

\title{
Whole-Genome Identification and Salt- and ABA-Induced Expression Trends of the Nicotiana tabacum CKX gene family
}

\author{
Wenwen Chen \\ Southwest University \\ Genhong Wang \\ Southwest University \\ Meiqin Yi \\ Southwest University \\ Chunman Song \\ China Tobacco Yunnan Industrial Corporation \\ Qian Gao \\ China Tobacco Yunnan Industrial Corporation \\ Qingyou Xia ( $\nabla$ xiaqy@swu.edu.cn) \\ Southwest University https://orcid.org/0000-0002-9263-2886 \\ Haiying Xiang \\ China Tobacco Yunnan Industrial Corporation
}

\section{Research Article}

Keywords: Cytokinin oxidase/dehydrogenase, Nicotiana tabacum, stress-induced expression, phylogenetic analysis

Posted Date: July 26th, 2021

DOI: https://doi.org/10.21203/rs.3.rs-731912/v1

License: (c) (i) This work is licensed under a Creative Commons Attribution 4.0 International License. Read Full License 


\section{Abstract}

Cytokinin hormones are indispensable for plant growth and development. Cytokinin oxidase/dehydrogenase (CKX) helps regulate the dynamic balance of endogenous cytokinin levels. However, little is known about the CKX genes of Nicotiana tabacum (NtCKXS). In silico analyses were used to isolate, characterize, and elucidate the phylogenetic relationships of 15 NtCKX genes. Multi-species phylogenetic tree construction placed NtCKX1-15 on five of the eight branches of the CKX phylogenetic tree. Protein structure and network analyses revealed that NtCKX genes located on the same phylogenetic branch generally contain several conserved motifs and have highly similar structures, with structural domains related to flavin adenine dinucleotide (FAD) and cytokinin-binding found on all of the predicted NtCKX-encoded proteins. The upstream promotor region of $N t C K X$ genes contained a large number of abiotic stress-responsive cis-acting elements, including DRE, ERE, MBS, MYB, and MYC. Gene expression analysis revealed that each NtCKX gene has a different response to salt- and exogenous ABA-stress. Four $N t C K X$ genes exhibited ABA-induced expression trends with varying peak times. Under salt-stress, NtCKX expression was significantly suppressed in two genes and upregulated in five others. In summary, we have provided basic in-formation about the CKX gene family of $N$. tabacum, and elucidated their gene expression patterns under abiotic stresses, including ABA- and salt-stress. The findings of this work can serve as a foundation for future study of the functions of $N$. tabacum CKX genes.

\section{Introduction}

Naturally occurring cytokinins are adenine derivatives with an isopentenyl side chain at the $N^{6}$ position. In combination with auxin and abscisic acid (ABA), cytokinins play an important role in plant growth and morphogenesis [1, 2]. Therefore, the dynamic homeostasis of endogenous cytokinin levels is critical for plants. Isopentyl transferases (IPTs) are responsible for the rate limiting step in cytokinin biosynthesis, and the two types of IPTs found in plants are tRNA-IPTs and adenylateIPTs $[3,4]$. In tRNAs that recognize codons beginning with $U$, the adenine residue immediately 3 ' to the anticodon may be modified by tRNA-IPT to have an isopentenyl- or cis-hydroxy isopentenyl- side chain at the $N^{6}$ position, thus forming ciszeatin (cZ) cytokinins [5]. Adenylate-IPTs may isopentenylate the $N^{6}$ position of adenine using dimethylallyl diphosphate as the side chain donor, to form $\mathrm{N}^{6}-\left(\Delta^{2}\right.$-isopentenyl)adenine (iP) or trans-zeatin (tZ) cytokinins [6]. Cytokinin dehydrogenases (CKXs) in particular play an important role in regulating cytokinin levels. CKXs are flavin adenine dinucleotide (FAD) oxidoreductases that cleave the $N^{6}$ sidechain of cytokinins to form adenine and the corresponding side chain aldehyde, thus inducing irreversible cytokinin degradation $[7,8]$.

The number of CKX genes identified in Arabidopsis thaliana, Zea mays, and Brassica rapa are 7, 10, and 12, respectively. The temporal and spatial expression pattern of CKX genes vary between different species. In $A$. thaliana, AtCKX1 is expressed in the shoots, lateral meristems, and roots. AtCKX5 is related to the development of pollen and axillary buds, whereas $A t C K X 6$ is expressed in the vascular systems of the cotyledons, leaves, and roots [7]. In Z. mays, ZmCKX1 is strongly expressed in the roots, and both $Z m C K X 2$ and $Z m C K X 12$ have been detected in the stem after germination [9]. In B. rapa, BrCKX 6 and $B r C K X 2-2$ are strongly expressed in the roots and gynoecium, respectively [10]. The functions of the CKX genes have also been extensively studied. The overexpression of AtCKX3 in A. thaliana can increase the growth rate of the primary roots and the length of the lateral and adventitious roots[7]. In Oryza sativa, a decrease in OsCKX2 expression can increase the number of reproductive organs, thus increasing the grain yield [11]. Overexpression of AtCKX1 in transgenic Nicotiana tabacum causes mature leaves to thicken and harden. Similarly, A. thaliana AtCKX3/AtCKX5 double mutants are known to produce more seedlings [12]. The silencing of the HvCKX1 gene decreases CKX enzyme level in Hordeum vulgare and leads to higher plant productivity [13].

Cytokinins also play an important role in plant stress responses. Vyroubalova et al. demonstrated that long-term salt-stress leads to the persistent and abundant expression of $Z m C K X 2, Z m C K X 3$, and $Z m C K X 6$ in $Z$. mays [14]. In the presence of drought-stress, induction of most Glycine max CKX genes occurs in the roots [15]. CK-deficient $A$. thaliana plants are known 
to exhibit a strong stress-tolerant phenotype [16]. Genetically modified $A$. thaliana plants that overexpress AtCKX3 have significantly greater root biomass than those of the wildtype $(60 \%)$, and this expanded root system increases the survival rate of the genetically modified line in drought conditions [17].

In this study, whole-genome sequencing was performed to identify and sequence the $C K X$ genes of $N$. tabacum. In addition, we analyzed the expression trends of $N t C K X$ genes under salt and exogenous ABA-stress. We have thus conducted a systematic study on the CKX genes of $N$. tabacum, which will lay the foundation for future study of their function.

\section{Materials And Methods}

\section{The identification and annotation of $\mathrm{N}$. tabacum NtCKX genes}

The genome sequences of $A$. thaliana [18], 0 . sativa [19], and Solanum lycopersicum [20] were retrieved from PlantGDB (http://www.plantgdb.org/ ), whereas the sequences of Arabidopsis lyrata, B. rapa, Populus trichocarpa and Physcomitrium patens were obtained from the study conducted by Liu et al. (2013). Basic Local Alignment Search Tool alignment was performed between the protein sequences of $A$. thaliana AtCKX and the $N$. tabacum genome

(https://solgenomics.net/organism/Nicotiana tabacum/genome), with an E-value of $1 \mathrm{e}^{-20}$. The predicted proteins of the resultant sequences were screened for the presence of FAD- and cytokinin-binding regions to obtain the candidate $N$. tabacum CKX gene sequences (Table S1). ProtComp 9.0 (http://linux1.sberry.com/berry.phtml/

topic $=$ protcomppl\&group $=$ programs\&subgroup $=$ proloc $)$ was used to predict the subcellular localization of NtCKX proteins, and the ExPASy ProtParam tool (https://web.

expasy.org/protparam/ ) was used to predict their isoelectric points and affinity coefficients.

\section{Multi-species phylogenetic analysis, multiple protein sequence alignment, and gene structure mapping}

A phylogenetic tree of the CKX gene families of N. tabacum, A. thaliana, O. sativa, B. rapa, P. trichocarpa, S. lycopersicum, and $P$. patens was created using MEGA 6.0 with the neighbor-joining ( $\mathrm{NJ}$ ) method with 1,000 bootstrap replications [21]. DNAMAN was used to investigate the conservativeness of the CKX gene family by analyzing the multiple sequence alignment between the CKX protein sequences of N. tabacum, A. thaliana, O. sativa, S. lycopersicum, and B. rapa [22]. The gene structure of the $N t C K X$ genes, including the number of introns and exons, and their genomic positions, were visualized using Gene Structure Display Server 2.0 (http://gsds.gaolab.

org/ )[23].

\section{Conserved protein motif analysis, cis-acting element analysis, and protein-protein inter-action network analysis}

MEME (http://alternate.meme-suite.org/tools/meme) and TBtools [24] were used to analyze the conserved protein motifs of the CKX genes. Descriptions of the cis-acting elements of the 15 NtCKX genes were obtained from PlantCARE (http://bioinformatics.psb.ugent.be/webtools

/plantcare/html/ ). The protein-protein interactions of the CKX genes of $A$. thaliana and $N$. tabacum were predicted using STRING (https://string-db.org/ ).

N. tabacum plant materials, the tissue-specific expression of NtCKX genes, and stress-induced expression trends

The N. tabacum plant materials were obtained from laboratory-grown $N$. tabacum K326. The seeds were disinfected using $75 \%$ alcohol for $10 \mathrm{~s}$, rinsed three times with sterile water, and then subjected to $10 \% \mathrm{NaOCl}$ for $6-8$ min to remove the seed coat. The seeds were washed another five times with sterile water before being cultured onto solid MS media. Cultivation was performed at $25^{\circ} \mathrm{C}$ with a $16 \mathrm{~h}: 8 \mathrm{~h}$ light/dark cycle. After two months of cultivation, the young leaves, stems, and roots 
were collected, along with the leaves, roots, stems, flowers and seeds of mature laboratory-grown N. tabacum plants. After collection, all the sample materials were immediately stored in liquid nitrogen and then transferred to a $-80^{\circ} \mathrm{C}$ freezer before measurements of gene expression levels. The stressed K326 plants were cultivated from seeds in soil at $25^{\circ} \mathrm{C}$ under $16 \mathrm{~h}: 8$ $\mathrm{h}$ light/dark cycles. At four-week-old, all of the leaves of each plant were sprayed with a $200 \mathrm{mM} \mathrm{NaCl}$ or a $100 \mu \mathrm{M} \mathrm{ABA}$ solution. Leaves from three biological replicates were then collected at $0 \mathrm{~h}, 3 \mathrm{~h}, 6 \mathrm{~h}, 12 \mathrm{~h}$, and $24 \mathrm{~h}$. All of the samples were immediately stored in liquid nitrogen after collection, and then transferred to a $-80^{\circ} \mathrm{C}$ freezer before being used for stressinduced expression profile analysis.

\section{RNA extraction and real-time reverse transcription polymerase chain reaction (RT-PCR) analysis}

RNA extraction was performed on all $N$. tabacum plant materials according to the Trizol reagent (Invitrogen) protocol. The GoScriptTM Reverse Transcription System (Promega) was used to reverse transcribe the extracted RNA to cDNA. Quantitative RT-PCR (qRT-PCR) was performed using a SYBR Premix Ex Taq TMIl kit (Takara, Japan) with specific quantitative PCR primers that were de-signed in PrimerQuest Tool (https://sg.idtdna.com/Primerquest/Home/Index ). NtEF1a was used as the internal control (Table S2). qTOWER 3.0 real-time PCR system (Analytik Jena AG, Jena, Germany) was used to measure gene expression levels in each tissue sample. Three biological replicates were collected for each treatment at each time point, and three technical replicates were used for each sample. The qRT-PCR regime consisted of an initial denaturation at $95^{\circ} \mathrm{C}$ for 3 minutes, followed by 40 cycles of $95^{\circ} \mathrm{C}$ for $10 \mathrm{~s}$ (denaturation) and $60^{\circ} \mathrm{C}$ for $1 \mathrm{~min}$ (annealing/elongation). The total duration of this procedure was $1 \mathrm{hr} 6 \mathrm{~min}$. Quantitative verification was performed using the $2^{-\Delta \Delta C t}$ method [25].

\section{Statistical Analysis}

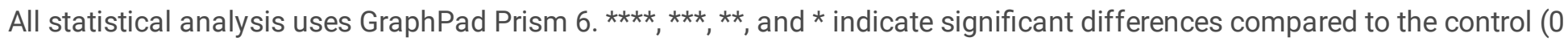
h) at $p<0.0001, p<0.001, p<0.01$, and $p<0.05$, respectively.

\section{Results}

\section{Identification and annotation of N. tabacum CKX genes}

Aligning the $A$. thaliana AtCKX sequences with the $N$. tabacum genome revealed $15 N$. tabacum NtCKX genes with similar structural domains. These genes were named NtCKX1-NtCKX15. The basic information of NtCKX gene family is presented in Table 1. The NtCKX gene family has between three-five exons, and the proteins they encode vary between 210-517 amino acids in length and 24,571.53-57,940.98 Da in molecular weight. All the proteins were hydrophilic, and their predicted isoelectric points ranged from 5.58 to 9.21. According to subcellular localization analysis of NtCKX gene expression, NtCKX1 was expressed in the cell membrane; NtCKX4-7, NtCKX10-12, and NtCKX14 were expressed in the extracellular matrix; $N t C K X 2, N t C K X 3$, and $N t C K X 8$ were expressed in the vacuoles; $N t C K X 13$ was expressed in the cytoplasm; and NtCKX15 was expressed in the chloroplasts.

\section{Phylogenetic tree, multiple protein sequence alignment, and gene structure analysis}

A phylogenetic tree was constructed using MEGA 6.0 for the CKX proteins of $N$. tabacum, $A$. thaliana, O. sativa, $B$. rapa, $P$. trichocarpa, S. lycopersicum, and P. patens to ascertain the evolutionary relationships of the CKX gene family (Fig. 1A). The phylogenetic tree has a total of eight branches, which are denoted as $a, \beta, \gamma, \delta, \varepsilon, \zeta, \eta$, and $\theta$. The NtCKX genes are mainly located on the $a, \beta, \delta, \zeta$, and $\theta$ branches. The a branch includes NtCKX1-3, NtCKX5-6 and NtCKX8; the $\beta$ branch includes $N t C K X 4$ and $N t C K X 7$; the $\delta$ branch includes NtCKX10-11; the $\zeta$ branch includes NtCKX 9 and NtCKX12; and the $\theta$ branch exclusively consists of the NtCKX13-15 genes. The $y$ branch consists of eight genes, which are the OsCKX3 and CKX genes of P. patens. 
Multiple protein sequence alignment analysis indicated that $N$. tabacum CKX genes exhibit a high degree of homology and conservativeness with respect to the CKX protein sequences of $A$. thaliana and $S$. lycopersicum (Figure S1). We found that genes located on the same branch have similar gene structures (Fig. 1B). For instance, NtCKX3, NtCKX5, NtCKX9 and $N t C K X 12$ have very similar gene structures, and all these genes have five exons that are flanked by four introns. NtCKX10 and $N t C K X 11$ have four exons and three introns, whereas $N t C K X 6$ and $N t C K X 8$ have three exons and two introns. NtCKX14 and NtCKX15 contain four exons and three introns. These NtCKX genes have the highest degree of intra-branch similarity in terms of structure and homology and have the same number of exons and introns. In contrast,NtCKX1, NtCKX2, NtCKX4, $N t C K X 7$, and NtCKX13 genes exhibit intra-branch differences in structure.

\section{Conserved protein motifs of the CKX genes of N. tabacum}

The MEME tool analysis of the conserved protein motifs of the CKX genes indicated that NtCKX genes on the same phylogenetic branch have highly similar motifs and that motifs $6,9,13,17$, and 20 are present in all the members of the NtCKX gene family (Fig. 2, Table S3). Except for NtCKX15, motifs 2, 4, and 15 are also present in all the members of this family. Motif 14 is present in members of the NtCKX gene family except for NtCKX genes on the a branch. Motifs 10 and 18 are also present in all NtCKX genes except for a-branch NtCKX genes and NtCKX4. Motifs 2 and 16 were annotated as motifs that could be related to the FAD-binding domain and motifs $3,5,6,7,13$, and 17 as possibly related to the cytokininbinding domain.

\section{Analysis of the protein-protein interaction network of N. tabacum and A. thaliana CKX genes}

Figure 3 illustrates the complex network of protein-protein interactions of the $N$. tabacum and $A$. thaliana CKX genes predicted using the STRING website. In A. thaliana, AtCKX1 has important functions in the development and

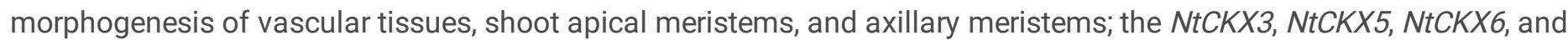
$N t C K X 8$ genes that correspond to AtCKX1 could have similar functions. The NtCKX9, NtCKX12, NtCKX13, and NtCKX14 genes that corresponded to $A t C K X 3$ may also autonomously regulate cytokinin levels and meristem cell viability. NtCKX4 and NtCKX7 may function similarly to $A t C K X 5$, which plays a role in regulating stem meristem differentiation and limiting the flow of cytokinins from the stem meristem. NtCKX1 and NtCKX2 could have the same functions as AtCKX6, and play a role in the development of cotyledon, leaf and root vascular systems, and stoma development in N. tabacum.

\section{Tissue expression profile of the NtCKX genes of $\mathrm{N}$. tabacum}

To study the functions of the NtCKX genes in further detail, RT-PCR was used to measure the expression of $N$. tabacum $C K X$ genes in young roots, leaves, and stems; and mature roots, leaves, stems, flowers, and seeds (Fig. 4). NtCKX11 was expressed in all tested tissues with high expression levels in mature roots. NtCKX4 was strongly ex-pressed in young stems, whereas NtCKX6 was expressed abundantly in seeds. NtCKX14 had high expression levels in seeds and mature leaves. $N t C K X 1$ was abundantly expressed in the flowers and mature roots, whereas NtCKX8 and NtCKX13 were strongly expressed in the seeds; these genes were either expressed at low or undetectable levels in all other tissues. NtCXK9 was abundantly expressed in young leaves, and it was also expressed in young roots and stems, mature stems, and seeds. $N t C X K 5$ was only expressed in mature leaves, whereas NtCKX 12 was only expressed in the seeds. NtCKX7 was specifically expressed in flowers and mature stems. The expression of $N t C K X 2,3,10$ and 15 was very low in all the sampled tissues and organs, so no data on its expression has been detected.

\section{Changes in the expression of N. tabacum NtCKX genes because of ABA- and salt-stress}

We also investigated the expression of the $N t C K X$ gene family in plants that were subjected to ABA- and salt-stress (Fig. 5). In the presence of ABA-stress, the expression of NtCKX4 was largely unchanged. The expression of NtCKX5, NtCKX7, $N t C K X 12, N t C K X 13$, and NtCKX15 fell to undetectable levels, and the changes in the expression levels of the other NtCKX genes was variable. For instance, the expression levels of $N t C K X 1, N t C K X 2$, and $N t C K X 6$ were suppressed at three hour, and 
then upregulated, with their expression levels peaking at $24 \mathrm{~h}$. The expression of NtCKX3 peaked at $6 \mathrm{~h}$ and then fell to undetectable levels. The expression levels of NtCKX8, NtCKX9 and NtCKX14 increased consistently up to $24 \mathrm{~h}$. The expression of $N t C K X 10$ resembled a parabolic curve; it gradually rose from $0 \mathrm{~h}$ to $12 \mathrm{~h}$ and then gradually decreased. The expression of $N t C K X 11$ was strongly upregulated at $3 \mathrm{~h}$ and then decreased gradually, returning to normal levels at $24 \mathrm{~h}$.

In salt-treated plants, NtCKX7, NtCKX12, NtCKX13 and NtCKX15 fell to undetectable levels. The expression of NtCKX4 was continuously down-regulation under salt treatment. The expression of $N t C K X 8$ did not change significantly, whereas $N t C K X 3$ expression was suppressed at $3 \mathrm{~h}$ but returned to normal levels shortly after. The expression of $N t C K X 2$ was suppressed starting from $12 \mathrm{~h}$. The expression levels of $N t C K X 1, N t C K X 10$, and NtCKX11 were parabola-like, as their expression was upregulated from the point of treatment until $6 \mathrm{~h}$, and then downregulated. The relative transcription levels of $N t C K X 9$ and $N t C K X 14$ gradually increased over time and peaked at 24 hour.

\section{Cis -acting elements in the promoter region of $\mathrm{N}$. tabacum NtCKX genes}

The promoter sequences of $N$. tabacum $C K X$ genes were analyzed to study their response to abiotic stress. The cis-acting elements in the promoter region of each NtCKX gene that were predicted to respond to environmental stress and hormones are shown in Table 2, with the exception of NtCKX14, whose promoter sequences could not be identified. These cis-acting elements include ABA-responsive elements (ABRE), drought-responsive elements (ERE, MBS, and MYB), and salt-responsive elements (DRE). MYC responds to induction by low temperatures, high salinity, and drought. ERE, MBS, and MYB respond to drought conditions and the accumulation of ABA. The promoter regions of NtCKX 8 and $N t C K X 9$ contained a large number of MYB elements. ERE was the most abundant element in the promoter region of NtCKX10, which also contained many MYB and MYC elements. The promoter region of NtCKX11 had more MYB elements than any other gene, and it also had a substantial number of ERE, MBS, MYC and DRE elements.

\section{Discussion}

Cytokinins are indispensable hormones for plant growth. CKX plays a critical role in the dynamic stabilization of endogenous cytokinin levels. Although the CKX gene family has been identified and studied in many plant species, the CKX genes of Solanaceae plants remain largely unexplored.

All flowering plants have undergone at least one whole-genome duplication (WGD) event in their evolutionary history [26]. This phenomenon likely arose as hybrids display enhanced adaptability to environmental changes, termed hybrid vigor [27]. Tobacco is a classic representative of Solanaceae plants. Common tobacco ( $N$. tabacum), in particular, is an allotetraploid plant that contains the full genomes of both of its ancestors, diploid $N$. sylvestris and $N$. tomentosiformis [28-30]. A. thaliana, A. lyrata, P. trichocarpa, S. lycopersicum, and O. sativa are all diploids, and their CKX gene families comprise nine, seven, nine, six, and ten members, respectively. The gene sequencing results of this study indicate that common tobacco contains 15 CKX gene family members. We speculate that WGD events account for the comparatively large number of CKX genes in N. tabacum. As WGD often leads to gene function redundancy, some of these genes may have been inactivated or differentiated [31, 32]. In this study, we have found that some genes belonging to the same phylogenetic branch generally have identical or similar sequences and structures, whereas most genes that belong to different branches have different structures and sequences. NtCKX13, NtCKX14, and NtCKX15 are located on the $\theta$ branch of the multi-species phylogenetic tree, and all three of these genes have similar gene structures and intact structural domains. In tissue expression and induced expression analysis, it was found that NtCKX13 and NtCKX14 are both strongly expressed in seeds and mature leaves and that NtCKX14 expression changes in response to abiotic stress. However, NtCKX15 is not expressed in any tissue or organ, with or without stress. We speculate that NtCKX15 may have been inactivated following WGD because of redundancy.

The tissue expression profile (Fig. 4) indicates that $N t C K X 11$ is expressed in all tissues, with especially high levels in mature roots. NtCKX1 is strongly expressed in flowers and mature roots, whereas NtCKX8 and NtCKX13 are strongly 
expressed in the seeds; these three genes are either unexpressed or expressed at low levels in all other tissues. The tissueand time-specific expression of these CKX genes is indicative of functional differentiation, resulting in each gene having a different regulatory role in plant development. Previous studies have shown that CKX genes are critical for root structure development [33]. For instance, the overexpression of AtCKX1, AtCKX2, and AtCKX3 increases initial root elongation and lateral root growth rates in $A$. thaliana [7]. Therefore, regulating the expression of $\mathrm{N}$. tabacum CKX genes may enhance root system development in these plants.

Cytokinin is one of the five major types of plant hormones. CKX plays an important role in biological adaption to environmental stress through its cytokinin degradation function $[14,15]$. Studies have shown that stress induction in $Z$. mays causes $Z m C K X 1$ to be strongly expressed in the leaves but not in the roots. Our study demonstrated that NtCKX genes have various roles in salt, low-temperature, and ABA-induced stress responses of $N$. tabacum. We found that each CKX gene had a different response to exogenous $A B A$, and expression levels in response to salt-stress were also varied. The cis-acting element analysis revealed that the NtCKX genes contained ABA-responsive, drought-responsive, and saltresponsive cis-acting elements. The ABRE element, in particular, is responsive to each of these stressors. Since ABRE is hypersensitive to salt stress and $A B A$, endogenous $A B A$ synthesis may decrease in response to the exogenous application of ABA. Concurrent regulation of endogenous hormones in the plant body will also lead to the accumulation of cytokinins, resulting in the inhibition of $N t C K X 4$ expression. CKX overexpressors generally exhibit a strong drought and salt tolerance phenotype, as well as enhanced heat-stress tolerance [34]. NtCKX 8 and NtCKX9 gene expression was strongly induced by $A B A$ and salt stress, which is consistent with the large number of MYB cis-acting elements in their promoter sequences. NtCKX10 and NtCKX11 both responded strongly to ABA and drought induction; this behavior may have been caused by the large number of $A B A$-, drought- and salt-responsive elements in their promoter regions. Some studies have shown that drought induction leads to the accumulation of ABA, which may enhance the tolerance of a plant to drought conditions [17, 35]. It has been demonstrated that this phenomenon arises from the regulation of the ABA-to-cytokinin ratio, which imbues the plant with enhanced drought and salt tolerance [16]. We hope that the findings of this study will serve as a reference for future studies of $N$. tabacum CKX genes and will guide efforts to engineer drought- or salinity-resistant plants via the modification of CKX genes.

\section{Conclusions}

A total of 15 NtCKX genes have been identified in this study, which are located on five of the eight phylogenetic branches of the CKX gene family. According to gene structure maps, conserved motif analysis, and multiple sequence alignment, CKX genes in the same phylogenetic branch usually have identical or similar gene structures, conserved motifs, and a high degree of homology. Most of the NtCKX genes contain many environmental stress-responsive elements in their upstream regulatory region, namely ABA-, drought-, and salt-responsive elements. The expression of each NtCKX gene varies both spatially and temporally, as well as their response to stress induction. In summary, this work has provided basic information about the CKX genes of $N$. tabacum, and it has established a foundation for future research into the functions of these genes.

\section{Abbreviations}

CKX Cytokinin oxidase/dehydrogenase

ABA abscisic acid

FAD flavin adenine dinucleotide

IPT Isopentyl transferases

cZ cis-zeatin cytokinins 


\section{Declarations}

Author Contributions WWC performed the experiments, analyzed the data, prepared figures and tables, authored drafts of the paper. GHW conceived and designed the experiments. MQY and CMS analyzed the data, contributed reagents/materials/analysis tools. QG analyzed the data, contributed reagents/materials/analysis tools. QYX and HYX authored or reviewed. All authors have read and agreed to the published version of the manuscript.

Funding This study was supported by grants from CTCCC (No. B20202NY1337) and CNTC research program (No.110202001020 (JY-03), No.110202001021 (JY-04), 110202001028 (JY-11) and 110201601034(JY-08)).

Data availability The data sets supporting the results of this article are included within the article and its additional file.

\section{Compliance with ethical standards}

Conflicts of interest The authors declare no competing interests.

\section{References}

1. Eklöf S, Åstot C, Blackwell J et al (1997) Auxin-Cytokinin Interactions in Wild-Type and Transgenic Tobacco. Plant Cell Physiol 38(3):225-235:11

2. Werner T, Motyka V, Strnad M (2001) Regulation of plant growth by cytokinin. Proceedings of the National Academy of Sciences 98:10487-10492

3. Kieber JJ, Schaller GE (2018) Cytokinin signaling in plant development. Development 145 DOI:10.1242/dev.149344

4. Polanska L, Vicankova A, Novakova M et al (2007) Altered cytokinin metabolism affects cytokinin, auxin, and abscisic acid contents in leaves and chloroplasts, and chloroplast ultrastructure in transgenic tobacco. J Exp Bot 58:637-649. DOI:10.1093/jxb/erl235

5. Yevdakova NA, Schwartzenberg K (2007) Characterisation of a prokaryote-type tRNA-isopentenyltransferase gene from the moss Physcomitrella patens. Planta 226:683-695. DOI:10.1007/s00425-007-0516-0

6. Lindner AC, Lang D, Seifert M et al (2014) Isopentenyltransferase-1 (IPT1) knockout in Physcomitrella together with phylogenetic analyses of IPTs provide insights into evolution of plant cytokinin biosynthesis. J Exp Bot 65:25332543. DOI:10.1093/jxb/eru142

7. Werner T, Motyka V, Laucou V et al (2003) Cytokinin-deficient transgenic Arabidopsis plants show multiple developmental alterations indicating opposite functions of cytokinins in the regulation of shoot and root meristem activity. Plant Cell 15:2532-2550. DOI:10.1105/tpc.014928

8. Kowalska M, Galuszka P, Frebortova J et al (2010) Vacuolar and cytosolic cytokinin dehydrogenases of Arabidopsis thaliana: heterologous expression, purification and properties. Phytochemistry 71:1970-1978.

DOI:10.1016/j.phytochem.2010.08.013

9. Zalabak D, Galuszka P, Mrizova K et al (2014) Biochemical characterization of the maize cytokinin dehydrogenase family and cytokinin profiling in developing maize plantlets in relation to the expression of cytokinin dehydrogenase genes. Plant Physiol Biochem 74:283-293. DOI:10.1016/j.plaphy.2013.11.020

10. Liu Z, Lv Y, Zhang M et al (2013) Identification, expression, and comparative genomic analysis of the IPT and CKX gene families in Chinese cabbage (Brassica rapa ssp. pekinensis). BMC Genom 14:594. DOI:10.1186/1471-2164-14594

11. Ashikari M, Sakakibara H, Lin S et al (2005) Cytokinin Oxidase Regulates Rice Grain Production. Research articles 309 
12. Bartrina I, Otto E, Strnad M et al (2011) Cytokinin regulates the activity of reproductive meristems, flower organ size, ovule formation, and thus seed yield in Arabidopsis thaliana. Plant Cell 23:69-80. DOI:10.1105/tpc.110.079079

13. Zalewski W, Galuszka P, Gasparis S et al (2010) Silencing of the HvCKX1 gene decreases the cytokinin oxidase/dehydrogenase level in barley and leads to higher plant productivity. J Exp Bot 61:1839-1851. DOI:10.1093/jxb/erq052

14. Vyroubalova S, Vaclavikova K, Tureckova V et al (2009) Characterization of new maize genes putatively involved in cytokinin metabolism and their expression during osmotic stress in relation to cytokinin levels. Plant Physiol 151:433447. DOI:10.1104/pp.109.142489

15. Le DT, Nishiyama R, Watanabe $Y$ et al (2012) Identification and expression analysis of cytokinin metabolic genes in soybean under normal and drought conditions in relation to cytokinin levels. PLoS One 7:e42411.

DOI:10.1371/journal.pone.0042411

16. Nishiyama R, Watanabe Y, Fujita Y et al (2011) Analysis of cytokinin mutants and regulation of cytokinin metabolic genes reveals important regulatory roles of cytokinins in drought, salt and abscisic acid responses, and abscisic acid biosynthesis. Plant Cell 23:2169-2183. DOI:10.1105/tpc.111.087395

17. Werner T, Nehnevajova E, Kollmer I et al (2010) Root-specific reduction of cytokinin causes enhanced root growth, drought tolerance, and leaf mineral enrichment in Arabidopsis and tobacco. Plant Cell 22:3905-3920.

DOI:10.1105/tpc.109.072694

18. Takei K, Sakakibara H, Sugiyama T (2001) Identification of genes encoding adenylate isopentenyltransferase, a cytokinin biosynthesis enzyme, in Arabidopsis thaliana. J Biol Chem 276:26405-26410.

DOI:10.1074/jbc.M102130200

19. Tsai YC, Weir NR, Hill K et al (2012) Characterization of genes involved in cytokinin signaling and metabolism from rice. Plant Physiol 158:1666-1684. DOI:10.1104/pp.111.192765

20. Matsuo S, Kikuchi K, Fukuda M et al (2012) Roles and regulation of cytokinins in tomato fruit development. J Exp Bot 63:5569-5579. DOI:10.1093/jxb/ers207

21. Tamura K, Stecher G, Peterson D et al (2013) MEGA6: Molecular Evolutionary Genetics Analysis version 6.0. Mol Biol Evol 30:2725-2729. DOI:10.1093/molbev/mst197

22. Julie D. Thompson TJG, Frédéric P, Jeanmougin F, Desmond G, Higgins (1997) The CLUSTAL_X windows interface: flexible strategies for multiple sequence alignment aided by quality analysis tools. Nucleic Acids Res 25:24

23. Hu B, Jin J, Guo AY et al (2015) GSDS 2.0: an upgraded gene feature visualization server. Bioinformatics 31:12961297. DOI:10.1093/bioinformatics/btu817

24. Chen C, Chen H, Zhang Y et al (2020) TBtools: An Integrative Toolkit Developed for Interactive Analyses of Big Biological Data. Mol Plant 13:1194-1202. DOI:10.1016/j.molp.2020.06.009

25. Livak KJ, Schmittgen TD (2001) Analysis of relative gene expression data using real-time quantitative PCR and the 2(Delta Delta C(T)) Method. Methods 25:402-408. DOI:10.1006/meth.2001.1262

26. Chen ZJ (2010) Molecular mechanisms of polyploidy and hybrid vigor. Trends Plant Sci 15:57-71. DOI:10.1016/j.tplants.2009.12.003

27. Proost S, Pattyn P, Gerats T et al (2011) Journey through the past: 150 million years of plant genome evolution. Plant $J$ 66:58-65. DOI:10.1111/j.1365-313X.2011.04521.x

28. Leitch IJ, Hanson L, Lim KY et al (2008) The ups and downs of genome size evolution in polyploid species of Nicotiana (Solanaceae). Ann Bot 101:805-814. DOI:10.1093/aob/mcm326

29. Kenton A, Parokonny AS, Gleba YY et al (1993) Characterization of the Nicotiana tabacum L. genome by molecular cytogenetics. Mol Gen Genet 240:159-169

30. Gazdova B, Siroky J, Fajkus J et al (1995) Characterization of a new family of tobacco highly repetitive DNA, GRS, specific for the Nicotiana tomentosiformis genomic component. Chromosome Res 3:245-254. 
DOI:10.1007/BF00713050

31. Rastogi S, Liberles DA (2005) Subfunctionalization of duplicated genes as a transition state to neofunctionalization. BMC Evol Biol 5:28. DOI:10.1186/1471-2148-5-28

32. Hahn MW (2009) Distinguishing Among Evolutionary Models for the Maintenance of Gene Duplicates. J Hered 100:605-617. DOI:10.1093/jhered/esp047

33. Reid DE, Heckmann AB, Novak O et al (2016) CYTOKININ OXIDASE/DEHYDROGENASE3 Maintains Cytokinin Homeostasis during Root and Nodule Development in Lotus japonicus. Plant Physiol 170:1060-1074.

DOI:10.1104/pp.15.00650

34. Mackova H, Hronkova M, Dobra J et al (2013) Enhanced drought and heat stress tolerance of tobacco plants with ectopically enhanced cytokinin oxidase/dehydrogenase gene expression. J Exp Bot 64:2805-2815.

DOI:10.1093/jxb/ert131

35. Huang X, Hou L, Meng J et al (2018) The Antagonistic Action of Abscisic Acid and Cytokinin Signaling Mediates Drought Stress Response in Arabidopsis. Mol Plant 11:970-982. DOI:10.1016/j.molp.2018.05.001

\section{Tables}

Table 1. Basic information of the cytokinin dehydrogenase/oxidase gene family of Nicotiana tabacum.

Table 2. Abiotic stress-responsive cis-acting elements in the promoter region of the NtCKX genes.

MYB: drought-responsive element; MYC: low-temperature, salt, and drought-responsive element; MBS: drought-responsive element; ABRE: ABA- responsive element; ERE: drought-responsive element; DRE: salt-responsive element.

\begin{tabular}{lllllll} 
Gene name & MYB & MYC & MBS & ABRE & ERE & DRE \\
\hline NtCKX1 & 7 & 5 & 1 & - & - & - \\
\hline NtCKX2 & 9 & 3 & - & 1 & 1 & - \\
\hline NtCKX3 & 8 & 4 & 1 & 2 & - & - \\
\hline NtCKX4 & 1 & 4 & - & 13 & - & 1 \\
\hline NtCKX5 & 1 & 1 & - & 1 & 3 & - \\
\hline NtCKX6 & 8 & 2 & - & 4 & 1 & - \\
\hline NtCKX7 & 8 & 4 & 2 & 8 & 1 & - \\
\hline NtCKX8 & 16 & 6 & 3 & 4 & - & - \\
\hline NtCKX9 & 10 & 1 & 1 & 4 & - & - \\
\hline NtCKX10 & 7 & 5 & 1 & 2 & 4 & \\
\hline NtCKX11 & 4 & 8 & 1 & 2 & 2 & 1 \\
\hline NtCKX12 & 4 & 3 & - & 4 & - & - \\
\hline NtCKX13 & 10 & 1 & 1 & - & 3 & 1 \\
\hline NtCKX15 & 10 & 3 & - & 6 & - & 1
\end{tabular}

"-" represented no cis-acting element in the promoter region of the NtCKX gene. 


\begin{tabular}{|c|c|c|c|c|c|c|c|}
\hline $\begin{array}{l}\text { Gene } \\
\text { name }\end{array}$ & Gene ID & $\begin{array}{l}\text { Protein } \\
\text { length } \\
\text { (aa) }\end{array}$ & $\begin{array}{l}\text { Molecular } \\
\text { weight } \\
\text { (Da) }\end{array}$ & $\begin{array}{l}\text { Theoretical } \\
\text { PI }\end{array}$ & $\begin{array}{l}\text { Grand } \\
\text { average of } \\
\text { hydropathicity } \\
\text { (GRAVY) }\end{array}$ & $\begin{array}{l}\text { Exon } \\
\text { number }\end{array}$ & $\begin{array}{l}\text { Subcellular } \\
\text { Location }\end{array}$ \\
\hline NtCKX1 & Nitab4.5_0000316g0350 & 375 & 41929.17 & 8.78 & -0.017 & 5 & $\begin{array}{l}\text { Cell } \\
\text { membrane }\end{array}$ \\
\hline $\mathrm{NtCKX2}$ & Nitab4.5_0008947g0010 & 352 & 39461.19 & 7.27 & -0.082 & 3 & Vacuole \\
\hline NtCKX3 & Nitab4.5_0000677g0110 & 445 & 49814.47 & 8.75 & -0.083 & 5 & Vacuole \\
\hline NtCKX4 & Nitab4.5_0001861g0120 & 416 & 45872.64 & 6.81 & -0.032 & 4 & $\begin{array}{l}\text { Extracell } \\
\text { matrix }\end{array}$ \\
\hline NtCKX5 & Nitab4.5_0000364g0020 & 373 & 41651.70 & 6.79 & -0.038 & 5 & $\begin{array}{l}\text { Extracell } \\
\text { matrix }\end{array}$ \\
\hline NtCKX6 & Nitab4.5_0001618g0190 & 331 & 37041.04 & 6.30 & -0.088 & 3 & $\begin{array}{l}\text { Extracell } \\
\text { matrix }\end{array}$ \\
\hline NtCKX7 & Nitab4.5_0005779g0060 & 482 & 54151.91 & 7.69 & -0.196 & 5 & $\begin{array}{l}\text { Extracell } \\
\text { matrix }\end{array}$ \\
\hline NtCKX8 & Nitab4.5_0000826g0120 & 311 & 34646.32 & 6.63 & -0.128 & 3 & Vacuole \\
\hline NtCKX9 & Nitab4.5_0008523g0060 & 493 & 55517.40 & 7.65 & -0.209 & 5 & $\begin{array}{l}\text { Extracell } \\
\text { matrix }\end{array}$ \\
\hline NtCKX10 & Nitab4.5_0000341g0010 & 517 & 57940.98 & 5.75 & -0.190 & 4 & $\begin{array}{l}\text { Extracell } \\
\text { matrix }\end{array}$ \\
\hline NtCKX11 & Nitab4.5_0000446g0210 & 517 & 57838.78 & 5.58 & -0.160 & 4 & $\begin{array}{l}\text { Extracell } \\
\text { matrix }\end{array}$ \\
\hline NtCKX12 & Nitab4.5_0000540g0320 & 451 & 51146.53 & 9.21 & -0.289 & 5 & $\begin{array}{l}\text { Extracell } \\
\text { matrix }\end{array}$ \\
\hline NtCKX13 & Nitab4.5_0010540g0020 & 474 & 53372.17 & 9.09 & -0.176 & 5 & Cytoplasm \\
\hline NtCKX14 & Nitab4.5_0010634g0030 & 379 & 42987.29 & 6.83 & -0.140 & 4 & $\begin{array}{l}\text { Extracell } \\
\text { matrix }\end{array}$ \\
\hline NtCKX15 & Nitab4.5_0007115g0010 & 210 & 24571.53 & 9.21 & -0.286 & 4 & Chloroplast \\
\hline
\end{tabular}

\section{Figures}




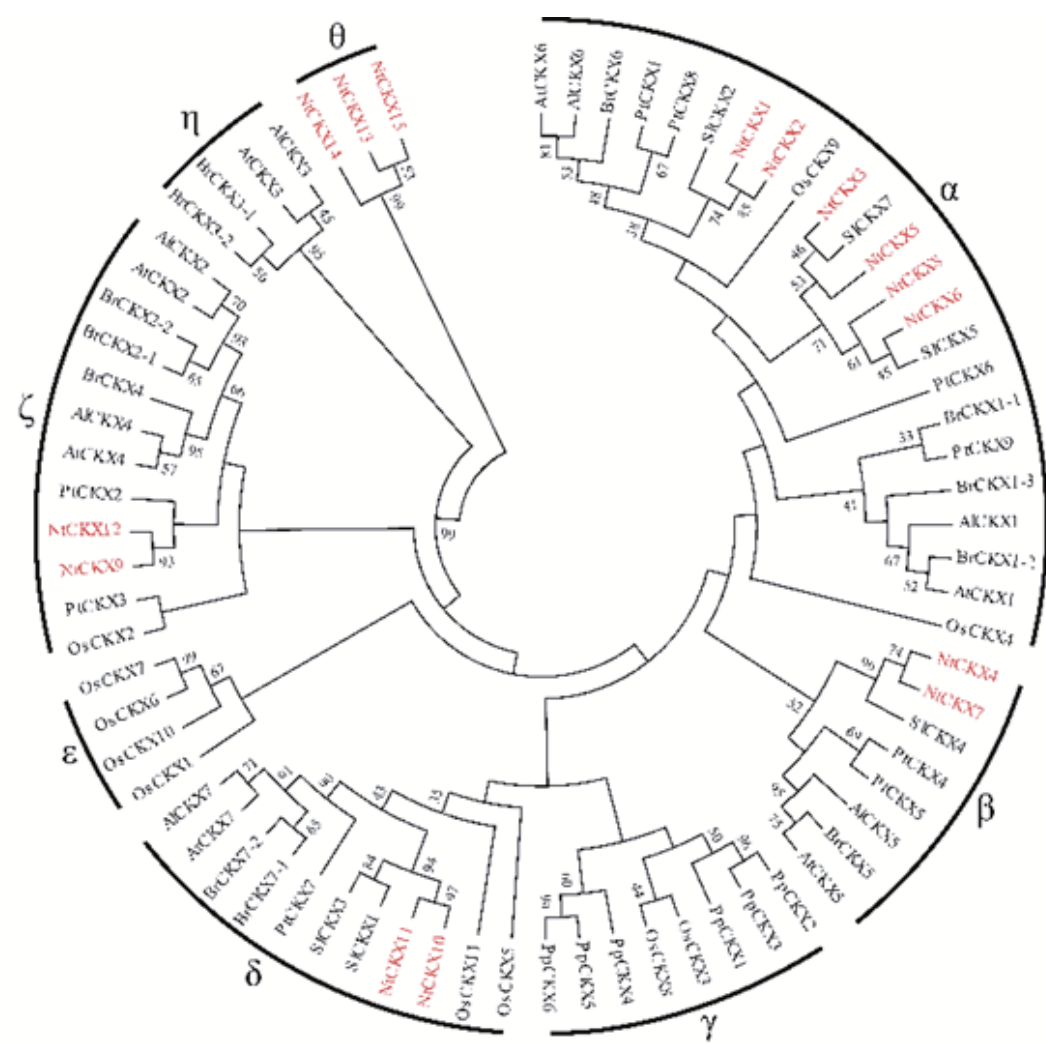

B

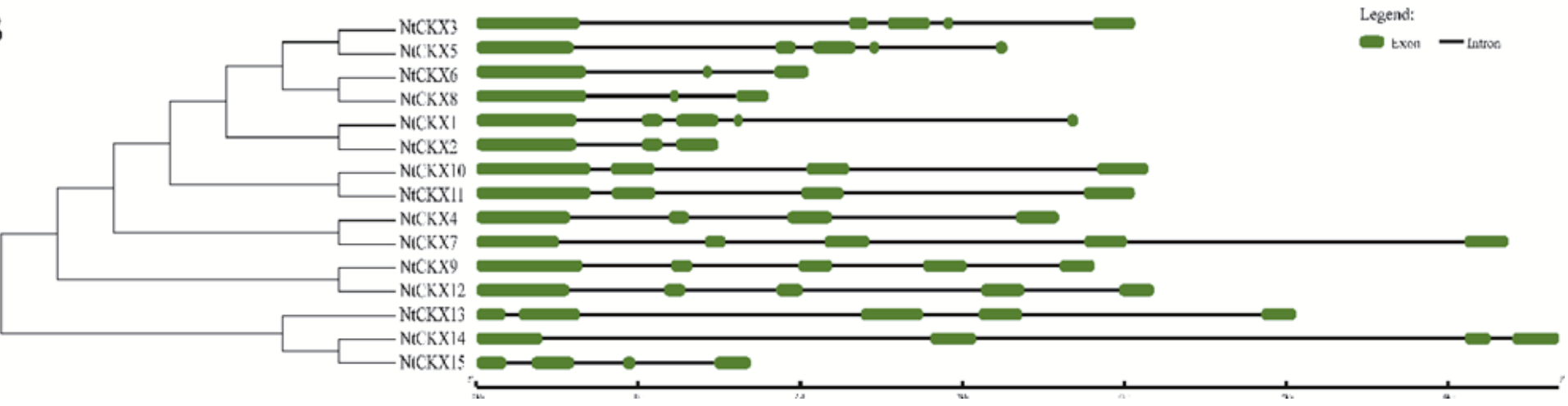

Figure 1

Phylogeny and gene structure of the N. tabacum CKX gene family. (A) Phylogenetic tree of the CKX gene family in N. tabacum and other species, including A. thaliana (At), A. lyrata (Al), B. rapa (Br), S. lycopersicum (SI), N. tabacum (Nt), 0. sativa (Os), and P. patens (Pp). The members of the N. tabacum CKX gene family are shown in red. (B) Phylogenetic tree and gene structure map (drawn to scale) of the NtCKX gene family. 


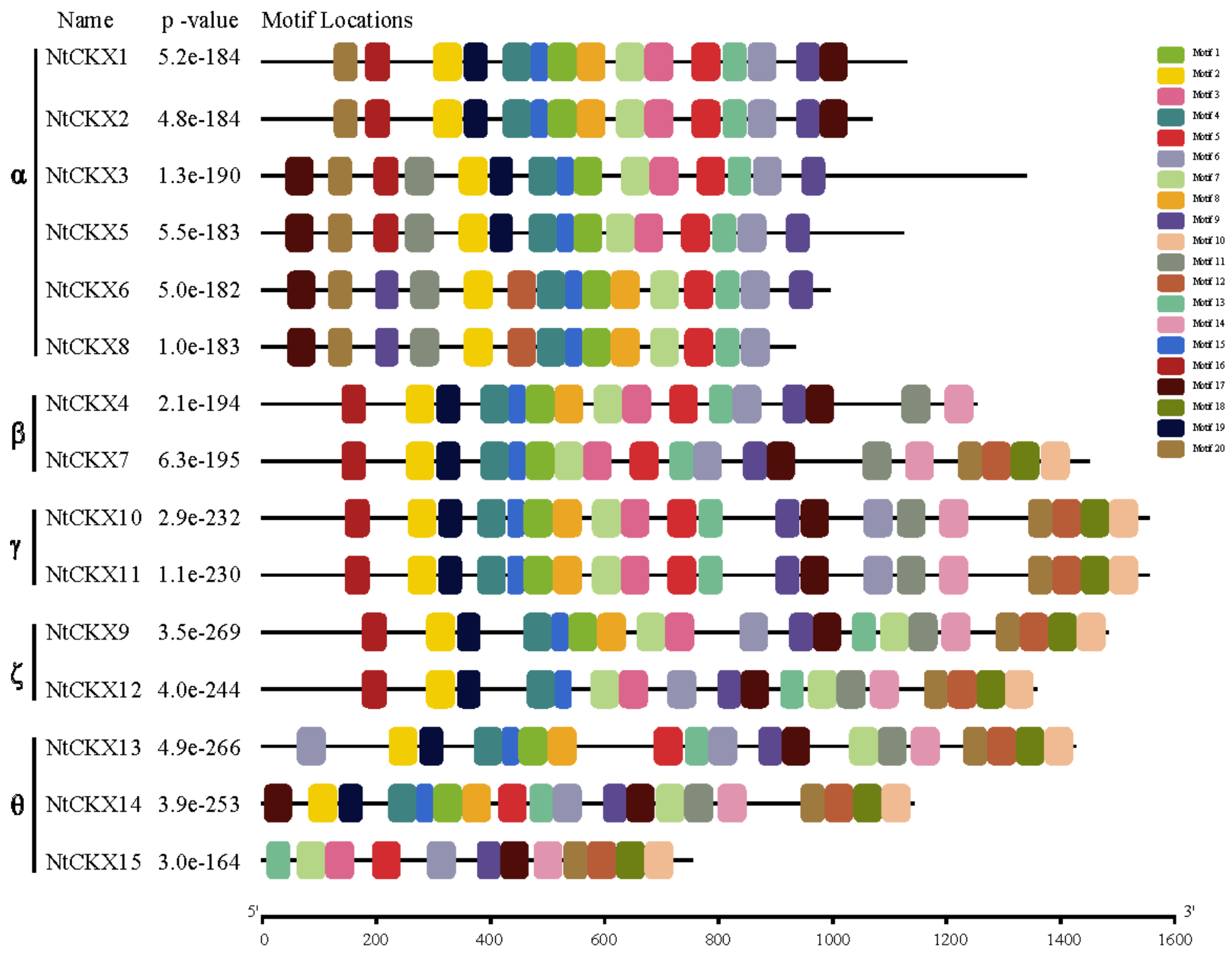

Figure 2

Conserved protein motif analysis of the NtCKX genes. Each color in the key (upper right corner) rep-resents a different conserved motif, which have been drawn according to their length. 


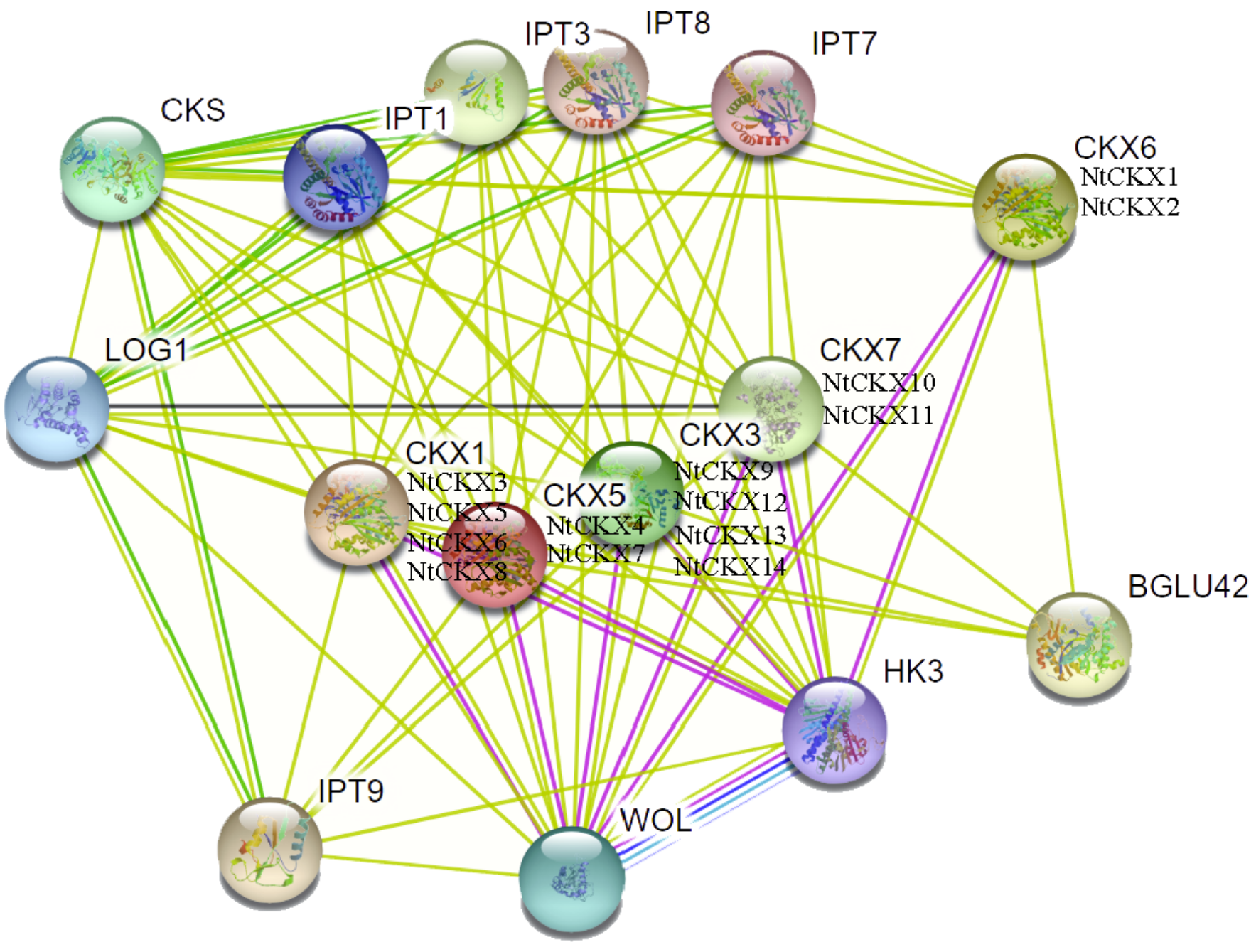

Figure 3

Protein-protein interaction network analysis of N. tabacum and A. thaliana CKX genes. Interac-tion map between the proteins coded by NtCKX and AtCKX genes. 

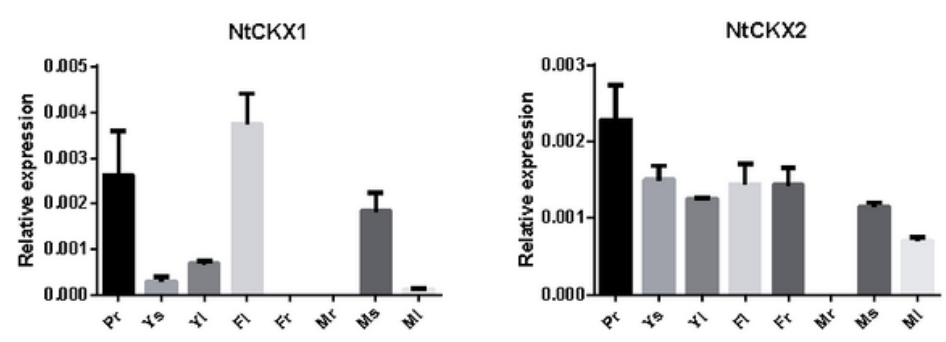

NtCKX5
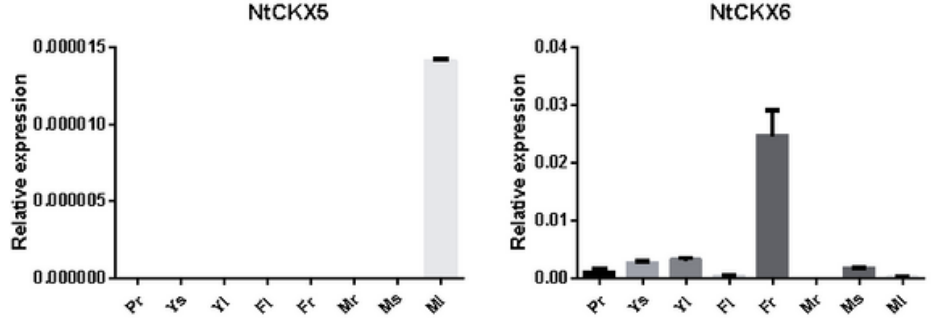

NtCKX9

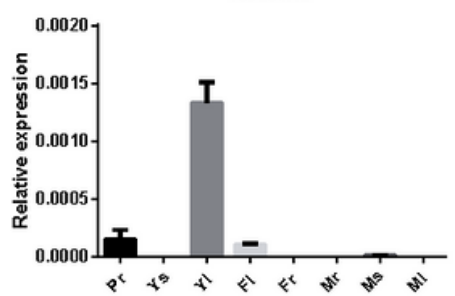

$\mathrm{NtCKX13}$

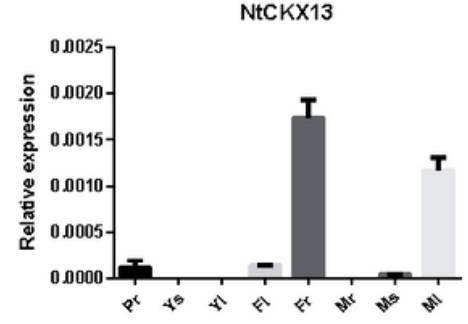

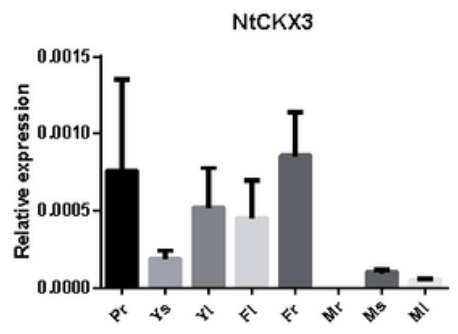

NtCKX7

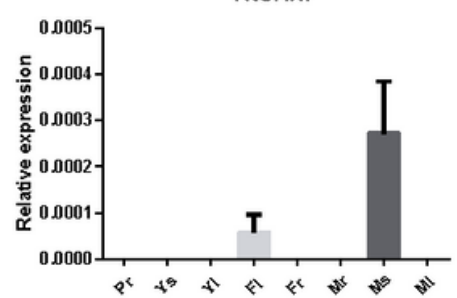

NtCKX11

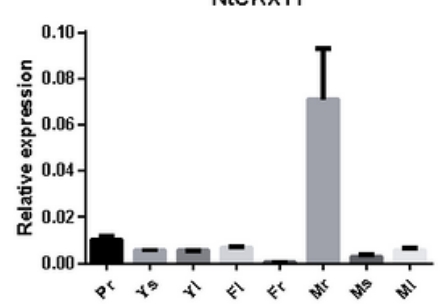

NtCKX4

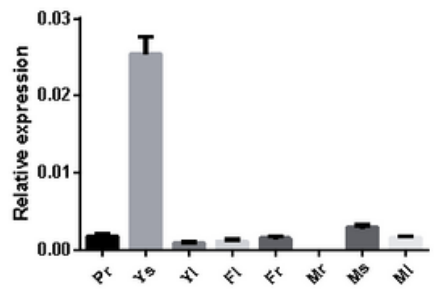

NtCKX8

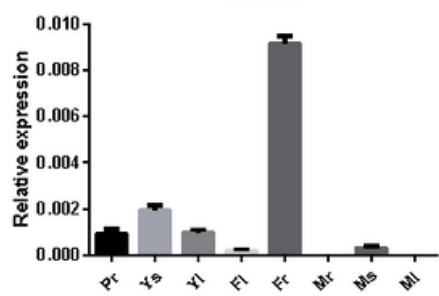

NtCKX12

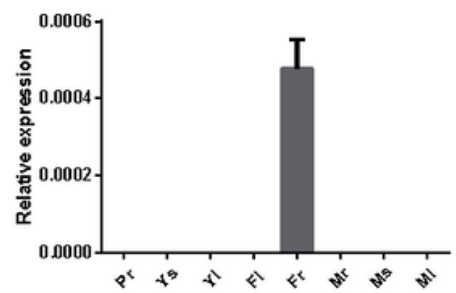

Figure 4

Tissue-specific expression of the NtCKX genes. Pr: young root; Ys: young stem; Yl: young leaves, Fl: flower, Fr: seedlings; Mr: Mature root; Ms: Mature stem; Ml: Mature leaves 
A
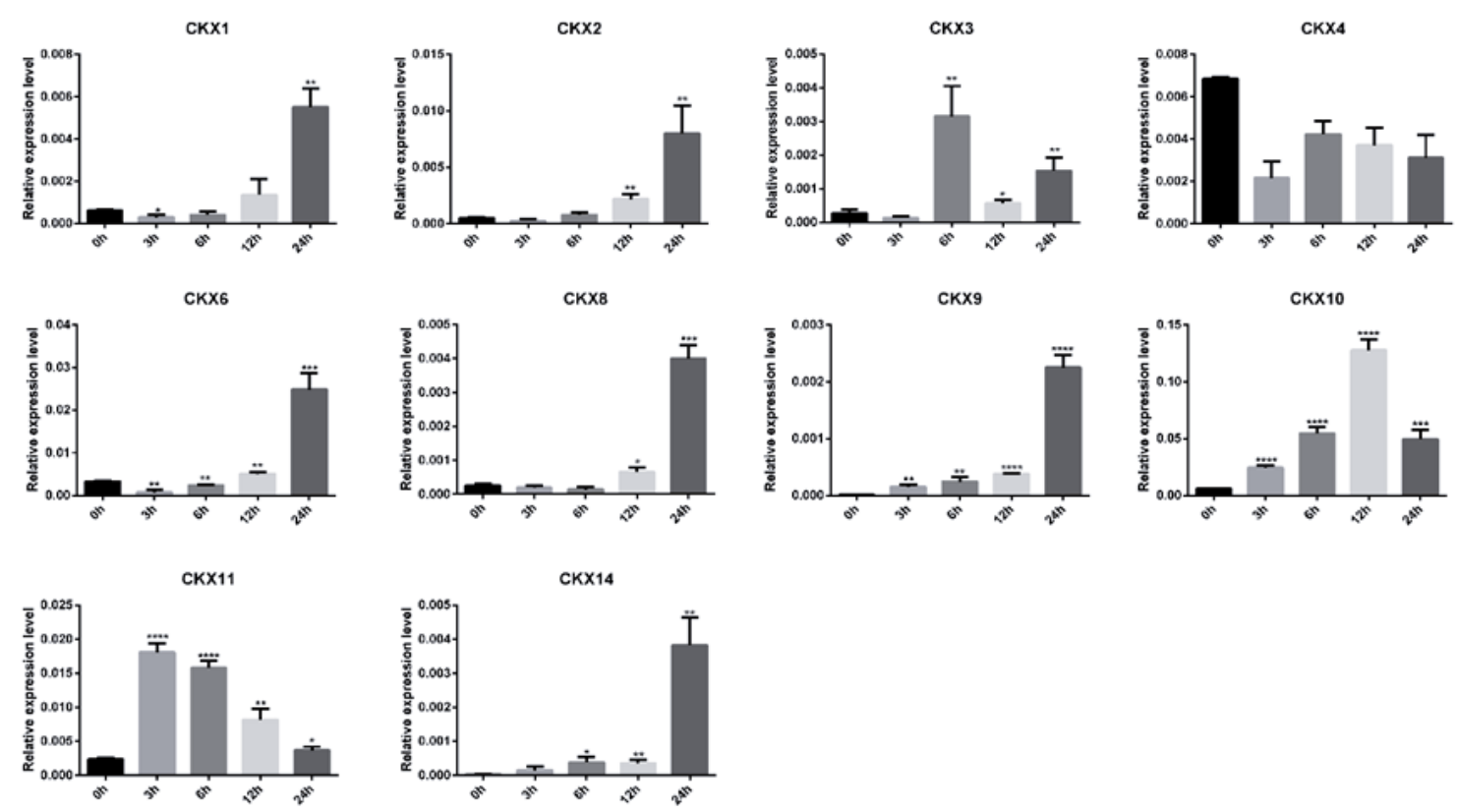

$\mathrm{B}$
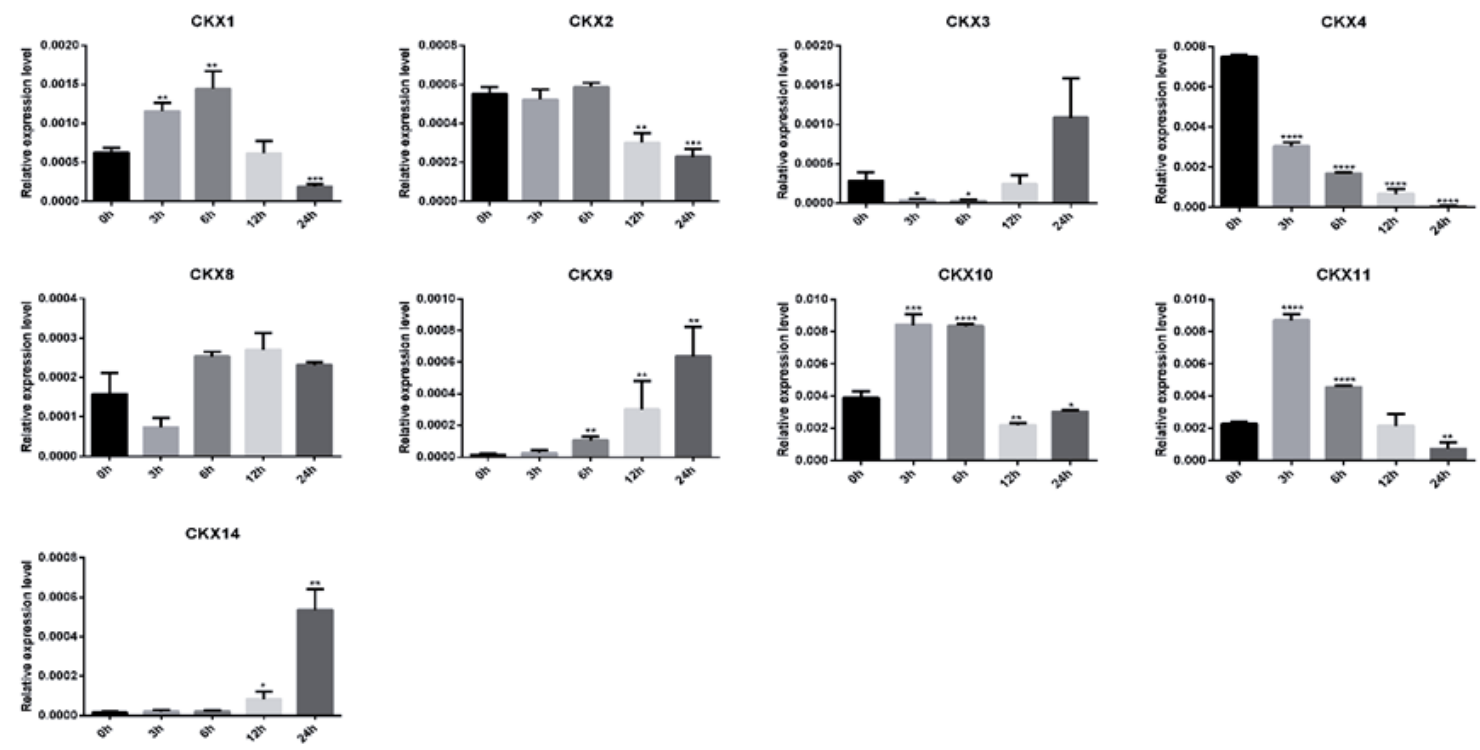

Figure 5

NtCKX expression in the presence of ABA and salt-stress. (A) NtCKX expression in the presence of ABA-stress. The indicated time points are $0 \mathrm{~h}, 3 \mathrm{~h}, 6 \mathrm{~h}, 12 \mathrm{~h}$, and $24 \mathrm{~h}$. The significance of the change in gene expression level with respect to the control at $0 \mathrm{~h}$ is designated as follows: ${ }^{\star \star \star *} \mathrm{p}<0.0001$, ${ }^{\star \star *} \mathrm{p}<0.001$, ${ }^{\star \star} p<0.01$, and ${ }^{*} \mathrm{p}<0.05$. (B) NtCKX expression in the presence of salt-stress. The indicat-ed time points are $0 \mathrm{~h}, 3 \mathrm{~h}, 6 \mathrm{~h}, 12 \mathrm{~h}$ and $24 \mathrm{~h}$. The significance of the

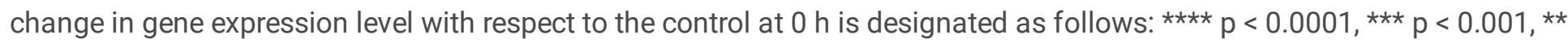
$p<0.01$, and $8 p<0.05)$.

\section{Supplementary Files}

This is a list of supplementary files associated with this preprint. Click to download.

- FigureS1.emf 
- Tables1.xlsx

- Tables2.xlsx

- Tables3.xlsx 